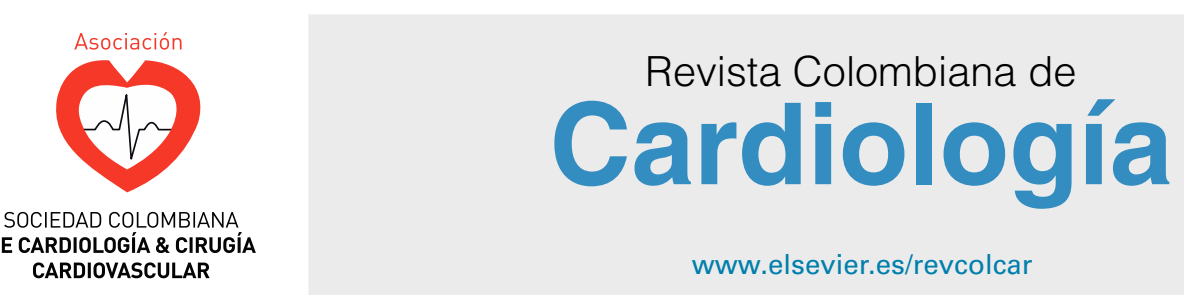

CARDIOLOGÍA DEL ADULTO - ARTÍCULO ORIGINAL

\title{
Caracterización de los pacientes con diagnóstico presuntivo de síndrome coronario agudo sin elevación del segmento ST que asisten a un servicio de urgencias
}

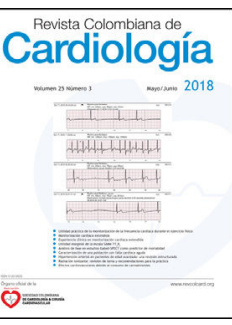

\author{
Luis Eduardo Silva Díaz-Granados*, Oscar Eduardo Sosa Mendoza, \\ Jorge A. Mahecha Bohórquez, Juan Pablo Polanco Cabrera, \\ Andrea C. Guechá Sánchez, Kimberly L. Horta Alfaro y Oscar A. Pacheco Jiménez
}

Departamento de Cardiología. Hospital Militar Central, Bogotá, Colombia

Recibido el 20 de octubre de 2016; aceptado el 18 de septiembre de 2017

Disponible en Internet el 7 de marzo de 2018

\section{PALABRAS CLAVE \\ Angina; \\ Infarto de miocardio; \\ Troponina T; \\ Angiografía coronaria}

\begin{abstract}
Resumen El dolor torácico es uno de los principales motivos de consulta al sistema de urgencias, y este es secundario a condiciones como el síndrome coronario agudo entre otras. Para su diagnóstico se requiere no sólo el resultado de la troponina, sino una evaluación clínica completa en la que se tengan en cuenta factores de riesgo cardiovascular, características del dolor y hallazgos electrocardiográficos. La interpretación inadecuada de las troponinas ultrasensibles lleva a que se someta al paciente a riesgos innecesarios a causa de estudios como el cateterismo cardiaco. Se llevó a cabo un estudio de corte trasversal en un hospital de Bogotá, en el que la causa principal de consulta en el servicio de urgencias fue el dolor torácico, y la primera causa de hospitalización en Cardiología el síndrome coronario agudo. Se recolectaron datos de 411 pacientes a quienes se les realizó arteriografía coronaria, la mayoría hombres con factores de riesgo como hipertensión arterial, tabaquismo y un evento coronario previo. Dentro de los estudios paraclínicos, la fracción de eyección promedio estuvo cercana al $50 \%$ y sólo en 201 pacientes se encontraron lesiones epicárdicas significativas. De la muestra analizada, el 13\% tenía creatinina mayor de $1,5 \mathrm{mg} / \mathrm{dl}$ como causa alterna de elevación del biomarcador y $28 \%$ tenía disfunción ventricular izquierda de algún grado. La mayoría de los pacientes en quienes no se documentaron lesiones angiográficamente significativas en el cateterismo cardiaco, la troponina no cumplía criterios de positividad con base en el aumento del $20 \%$ respecto al valor inicial si este era positivo o de $50 \%$ en caso de que el primer valor fuese negativo. Adicionalmente, del grupo de pacientes con enfermedad coronaria angiográficamente significativa fue más frecuente la combinación de tres o más factores de riesgo cardiovascular en presencia de biomarcador positivo.

(c) 2018 Sociedad Colombiana de Cardiología y Cirugía Cardiovascular. Publicado por Elsevier España, S.L.U. Este es un artículo Open Access bajo la licencia CC BY-NC-ND (http:// creativecommons.org/licenses/by-nc-nd/4.0/).
\end{abstract}

\footnotetext{
* Autor para correspondencia.

Correo electrónico: lesilvadg@gmail.com (L.E. Silva Díaz-Granados).
} 


\section{KEYWORDS}

Angina;

Myocardial infarction;

Troponin T;

Coronary angiography
Profile of patients with non-ST elevation acute coronary syndrome that attend an Emergency Department

Abstract Chest pain is one of the main reasons for consulting the Emergency Department, and it is secondary to conditions, such as acute coronary syndrome. For its diagnosis, it not only requires a Troponin result, but also a full clinical evaluation, in which factors like cardiovascular risk have to be taken into account, as well as characteristics of the pain and the findings on the electrocardiogram. The poor interpretation of the ultrasensitive Troponins leads to the patient being subjected to unnecessary risks due to studies such as cardiac catheterisation. A cross-sectional study was conducted in a hospital in Bogota, in which chest pain was the main reason for consulting the Emergency Department, and acute coronary syndrome the first cause of admission to Cardiology. The study included a total of 411 patients on whom a coronary angiography was performed. The majority were males with risk factors such as arterial hypertension, smokers, and with a previous coronary event. Among the para-clinical studies, the mean ejection fraction was around $50 \%$, and significant epicardial lesions were found in only 201 patients. Of the sample analysed, $13 \%$ had a creatinine greater than $1.5 \mathrm{mg} / \mathrm{dl}$ as an alternative cause of the elevation of the biomarker, and $28 \%$ had some degree of left ventricular function. the majority of patients that did not have significant angiographic lesions in the cardiac catheterisation documented, the Troponin did not meet the criteria for being positive, based on an increase of $20 \%$ as regards the initial value if this was positive or $50 \%$ in the case where the first value was negative. Furthermore, of the patient group with significant angiographic coronary disease, the combination of three or more cardiovascular risk factors was the most frequent in the presence of a positive biomarker.

(c) 2018 Sociedad Colombiana de Cardiología y Cirugía Cardiovascular. Published by Elsevier España, S.L.U. This is an open access article under the CC BY-NC-ND license (http:// creativecommons.org/licenses/by-nc-nd/4.0/).

\section{Introducción}

El dolor torácico es la causa de 8 a 10 millones de visitas al sistema de urgencias en Estados Unidos; de los pacientes que ingresan con este síntoma, menos del $25 \%$ son diagnosticados con un síndrome coronario agudo ${ }^{1,2}$. Entre 5 y $10 \%$ de los pacientes con dolor torácico pueden ser dados de alta cursando con un infarto de miocardio, de ahí que se deba dar el enfoque adecuado para evitar errores en el manejo $0^{3,4}$.

Para el médico que trabaja en el servicio de urgencias el reto es identificar con exactitud a los pacientes con dolor torácico que amenaza la vida y quiénes pueden recibir tratamiento ambulatorio. Se deben identificar tres grupos principales: síndrome coronario agudo, causas cardíacas diferentes a síndrome coronario agudo y causas no cardíacas $^{3,4}$.

No hay una prueba perfecta que tenga la mejor sensibilidad y especificidad para el diagnóstico del infarto agudo del miocardio, por ende es vital el juicio clínico para determinar la probabilidad de isquemia miocárdica y la estratificación del riesgo de muerte o infarto no fatal a corto plazo ${ }^{3}$.

La troponina es una proteína globular de gran tamaño; contiene tres subunidades polipeptídicas: troponina c (fijadora de calcio), troponina I (inhibidora de la interacción actina-miosina) y troponina $\mathrm{T}$ (fijadora de tropomiosina). Cuando hay necrosis de las células del tejido miocárdico, se pierde la integridad de la membrana celular, fenómeno que hace que el contenido intracelular se difunda hacia la circulación periférica elevándose el marcador sérico, permitiéndole al clínico hacer diagnósticos presuntivos de lesión miocárdica ${ }^{5,6}$.
Con el paso del tiempo, los biomarcadores en cardiología han presentado una evolución significativa. Las pruebas iniciales no tenían un rendimiento operativo adecuado, por tanto evolucionaron a pruebas que incrementaban su sensibilidad y que obligaron al médico a realizar un estudio juicioso basado en una historia clínica completa y una estratificación mediante escalas de riesgo clínico, aumentándose así la probabilidad de que el resultado del biomarcador concordara con el diagnóstico efectuado de forma clínica y presuntiva $^{5-8}$.

Tras años de evolución, se dispone de la troponina ultrasensible que, si bien no es el biomarcador que cumple las características ideales, permite un diagnóstico más temprano; por consiguiente su uso es universal y así se ha postulado en las guías tanto para diagnóstico como para estratificación del riesgo de mortalidad en síndrome coronario agudo. Según la tercera definición de infarto agudo de miocardio, tanto de las guías europeas de cardiología como las norteamericanas, el diagnóstico de trabajo de síndrome coronario agudo depende de la clínica sugestiva de enfermedad coronaria, asociada a biomarcadores ultrasensibles positivos con un incremento o descenso del $20 \%$, configurando el diagnóstico y por ende, haciendo imperativo el cateterismo cardiaco como método invasivo para estratificar la enfermedad y poder brindar tratamiento oportuno ${ }^{9,14}$.

Infortunadamente el desconocimiento de las características propias del biomarcador y de las campañas internacionales que inducen al clínico a sospechar el diagnóstico de enfermedad coronaria aguda, incluso en ausencia de datos en la historia clínica, ha hecho que la prueba se use de forma indiscriminada y que su interpretación 
muchas veces se aleje del contexto clínico individualizado y real $^{15-17}$.

En publicaciones que datan del año 2007, hechas en revistas de alto impacto, el biomarcador propiamente dicho adquiere un papel protagónico en el escenario de toma de decisiones, en tanto que el juicio clínico está siendo relegado a un segundo plano. La innovación de los biomarcadores cardiacos descritos como ultrasensibles, sugiere un alto valor predictivo negativo $\mathrm{y}$, en ausencia de un criterio clínico adecuado, el valor predictivo positivo no supera el $60 \%$, dependiendo del fabricante; por esto aún sigue siendo indispensable el juicio clínico de acuerdo con las características del cuadro y los factores de riesgo de cada paciente ${ }^{18-21}$.

\section{Metodología}

Se llevó a cabo un estudio descriptivo de corte trasversal en el que se incluyeron beneficiarios del subsistema de salud de las fuerzas militares, que ingresaron por dolor torácico al servicio de urgencias y recibieron diagnóstico presuntivo de síndrome coronario agudo sin elevación del segmento ST desde el $1^{\circ}$. de enero de 2013 hasta el 31 diciembre de 2014. El diagnóstico de síndrome coronario agudo se basó en la presencia de dolor torácico asociado a cambios electrocardiográficos y elevación o no de biomarcadores.

Se hizo un muestreo por conveniencia en el cual se incluyeron mayores de 18 años con diagnóstico clínico de síndrome coronario agudo sin elevación del segmento ST (SCASEST) con biomarcadores de lesión miocárdica (troponina ultrasensible T) durante el periodo del estudio, según la definición última y vigente de infarto agudo de miocardio.

\section{Criterios de selección}

Se incluyeron pacientes con edad mayor de 18 años y diagnóstico presuntivo de SCASEST quienes ingresaron por el servicio de urgencias del HCM durante el periodo definido del estudio, con disponibilidad del reporte de troponina T ultrasensible, y a quienes se les hubiera realizado el cateterismo cardiaco en dicha institución.

Se excluyeron pacientes llevados a procedimiento de cateterismo cardiaco para medición de reserva de flujo fraccional a fin de determinar lesiones significativas, aquellos a quienes que se les hiciera el procedimiento fuera del escenario del SCASEST, o cateterismo cardiaco por trombólisis fallida y aquellos con diagnóstico de síndrome coronario agudo con elevación del segmento ST.

\section{Resultados}

Se hizo la recolección y diligenciamiento de una base de datos a partir de la cual se obtuvieron los resultados de cateterismo cardiaco realizado en la institución en el periodo de tiempo establecido. Para el tiempo del estudio se practicaron 874 procedimientos; sin embargo, se excluyeron 461 pacientes que no cumplieron los criterios de selección e ingresaron 411.

Respecto a la caracterización de estos pacientes, la mayoría eran hombres $(65,45 \%)$ y las comorbilidades más frecuentes fueron hipertensión arterial $(71,53 \%)$, infarto
Tabla 1 Características iniciales de los pacientes

\begin{tabular}{|c|c|}
\hline Característica & $\mathrm{n}=411$ \\
\hline Mujeres, no. (\%) & $142(34,55)$ \\
\hline \multicolumn{2}{|l|}{ Comorbilidades } \\
\hline Hipertensión arterial, no. (\%) & $294(71,53)$ \\
\hline IAM previo, no. (\%) & $181(44,04)$ \\
\hline Tabaquismo, no. (\%) & $130(31,63)$ \\
\hline Dislipidemia, no. (\%) & $112(27,25)$ \\
\hline Diabetes mellitus, no. (\%) & $109(26,52)$ \\
\hline Angina, no. (\%) & $81(19,71)$ \\
\hline Historia familiar, no. (\%) & $26(6,33)$ \\
\hline Accidente cerebrovascular, no. (\%) & $12(2,92)$ \\
\hline \multicolumn{2}{|l|}{ Tiempo desde el inicio del dolor } \\
\hline De 1 a 3 horas, no. (\%) & $73(17,76)$ \\
\hline De 3 a 6 horas, no. (\%) & $58(14,11)$ \\
\hline De 6 a 12 horas, no. (\%) & $65(15,81)$ \\
\hline Mayor a 12 horas, no. (\%) & $215(52,31)$ \\
\hline \multicolumn{2}{|l|}{ Características clínicas } \\
\hline Dolor torácico típico, no. (\%) & $239(58,15)$ \\
\hline $\begin{array}{l}\text { Presión arterial sistólica en } \mathrm{mm} \mathrm{Hg} \text {, } \\
\text { mediana }(\mathrm{Q} 1, \mathrm{Q} 3)\end{array}$ & $135(122-152)$ \\
\hline $\begin{array}{l}\text { Presión arterial diastólica en mm } \\
\mathrm{Hg} \text {, mediana }(\mathrm{Q} 1, \mathrm{Q} 3)\end{array}$ & $78(70-89)$ \\
\hline \multicolumn{2}{|l|}{ Resultados paraclínicos } \\
\hline $\begin{array}{l}\text { Creatinina en } \mathrm{mg} / \mathrm{dl} \text {, mediana }(\mathrm{Q} 1 \text {, } \\
\text { Q3) }\end{array}$ & $0,92(0,75-1,18)$ \\
\hline $\begin{array}{l}\text { Troponina primer valor positivo, } \\
\text { no. (\%) }\end{array}$ & $301(73,23)$ \\
\hline $\begin{array}{l}\text { Troponina segundo valor positivo, } \\
\text { no. (\%) }\end{array}$ & $308(74,94)$ \\
\hline $\begin{array}{l}\text { Evidencia de lesiones epicárdicas } \\
\text { en cateterismo, no. (\%) }\end{array}$ & $201(48,91)$ \\
\hline
\end{tabular}

agudo de miocardio previo $(44,04 \%)$ y tabaquismo $(31,63 \%)$. Sólo 81 pacientes reportaron cuadros de angina previos y 26 refirieron antecedente familiar de enfermedad coronaria. El 52,31\% reportó una duración del dolor torácico mayor a 12 horas, 239 presentaron dolor torácico típico y presión arterial tanto sistólica como diastólica dentro del rango de normalidad en la mayoría de estos. En la tabla 1 se resumen el resto de variables de caracterización de los pacientes.

En cuanto a paraclínicos, la mediana de creatinina fue normal $(0,92 \mathrm{mg} / \mathrm{dl})$ y la distribución de la fracción de eyección del ventrículo izquierdo medida por ecocardiograma durante la hospitalización en promedio fue de 49,11\%; más del $55 \%$ de los pacientes tuvieron una FEVI mayor al 50\%, con valor mínimo reportado de $14 \%$ y máximo de $68 \%$ (fig. 1 ). Según el punto de corte de la institución, el valor de la troponina inicial fue positivo en 301 pacientes así como también lo fue el segundo valor de troponina en $74,94 \%$ de los pacientes evaluados. De los cateterismos cardiacos, el $48,91 \%$ de los pacientes tuvo lesiones en arterias epicárdicas durante el estudio.

En cuanto a los pacientes cuyo cateterismo cardiaco no demostró enfermedad epicárdica significativa, el $27 \%$ no tenía segunda toma de troponina, hecho que llevó a la estratificación invasiva de pacientes que incluso tenían el primer biomarcador negativo. Otro punto interesante es que el $26 \%$ 


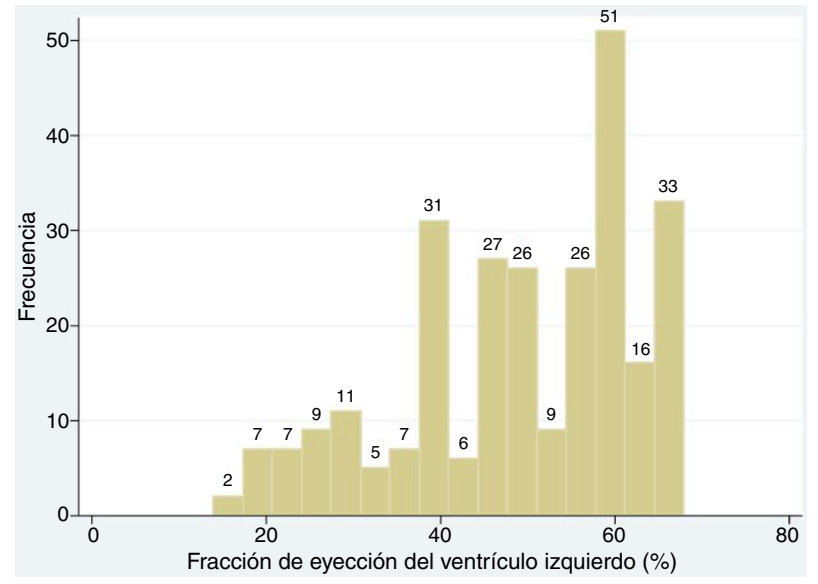

Figura 1 Histograma que muestra la distribución de la fracción de eyección del ventrículo izquierdo.

de pacientes tenían curva, tanto significativa como no significativa, en descenso; del subgrupo de pacientes que tenían el primer valor positivo, $32 \%$ hicieron delta superior a $20 \%$; sin embargo, de esos, 37\% tenían más de 6 horas desde el inicio de los síntomas por lo cual el primer resultado era diagnóstico. Si bien el diagnóstico de síndrome coronario agudo es eminentemente clínico, el 58,15\% quedó registrado por presentar dolor torácico de características típicas y en $17 \%$ de los casos había causas alternas de elevación de troponina como en pacientes con crisis hipertensiva.

Al remitirnos a los antecedentes que pueden contribuir al incremento de la probabilidad pretest del síndrome coronario agudo, la presencia de evento coronario previo se encontraba en $44,04 \%$ de los pacientes y el $13 \%$ de los casos tenían la creatinina mayor de $1,5 \mathrm{mg} / \mathrm{dl}$ como causa alterna de elevación del biomarcador y $28 \%$ tenían disfunción ventricular izquierda de algún grado.

Se recolectó información acerca de los hallazgos electrocardiográficos y ecocardiográficos de los pacientes con SCASEST sin lesiones epicárdicas en el cateterismo cardiaco. Se revisó la información del reporte del electrocardiograma de 194 pacientes, de los cuales 46 no reportó alteraciones, $10,82 \%$ reportó trastornos inespecíficos de la repolarización y cerca del $20 \%$ tenían algún signo de isquemia o de lesión miocárdica. No fue muy frecuente el reporte de arritmias ni bloqueos de rama (tabla 2).

Adicionalmente se revisaron los ecocardiogramas de este mismo subgrupo de pacientes y se logró tener disponible la información de 162 de los cuales el 48,15\% tenía algún trastorno de la contractilidad; 5 fue la mediana de segmentos comprometidos, sólo se documentó un caso de trombo intracavitario y 77 casos tenían algún grado de disfunción diastólica (tabla 2).

Al analizar cada factor de riesgo y su prevalencia según los hallazgos en el cateterismo cardiaco (lesiones epicárdicas vs. sin lesiones epicárdicas), el único factor con una diferencia estadísticamente significativa al comparar ambos grupos fue el antecedente de angina con una razón de prevalencia de 0,51 (IC 95\% 0,29-0,87, valor de p=0,008). Al comparar los otros factores de riesgo no hubo diferencias entre ambos grupos, tal como se muestra en la tabla 3.
Tabla 2 Características de los paraclínicos - Pacientes con SCASEST sin lesiones epicárdicas

\begin{tabular}{ll}
\hline Electrocardiograma & $\mathrm{n}=194$ \\
\hline Sin alteraciones, no. (\%) & $46(23,71)$ \\
Signos de isquemia, no. (\%) & $37(19,07)$ \\
Signos de lesión, no. (\%) & $38(19,59)$ \\
Signos de necrosis, no. (\%) & $21(10,82)$ \\
Bloqueo de rama izquierda, no. (\%) & $16(8,25)$ \\
Bloqueo de rama derecha, no. (\%) & $18(9,28)$ \\
Taquiarritmia, no. (\%) & $17(8,76)$ \\
Bradiarritmia, no. (\%) & $10(5,18)$ \\
Trastorno inespecífico de la & $21(10,82)$ \\
$\quad$ repolarización, no. (\%) & \\
Ecocardiograma transtorácico & $\mathrm{n}=162$ \\
Trastorno de la contractilidad, no. (\%) & $78(48,15)$ \\
Segmentos comprometidos, mediana & $5(3,6)$ \\
$\quad$ Q1, Q3) & \\
Disfunción diastólica, no. (\%) & $77(47,53)$ \\
Trombo intracavitario, no. (\%) & $1(0,62)$ \\
\hline
\end{tabular}

\section{Discusión}

No existe un biomarcador que tenga una sensibilidad y especificidad perfecta para el diagnóstico de síndrome coronario agudo. Históricamente se han empleado diferentes paraclínicos que buscan incrementar el valor predictivo ya sea positivo o negativo que tienen el abordaje clínico y el electrocardiograma en el servicio de urgencias ${ }^{2}$.

La evaluación del paciente con dolor torácico es uno de los mayores retos para los médicos que trabajan en los servicios de urgencias dado que es una de las principales causas de consulta en algunas instituciones como la nuestra. Tanto el diagnóstico como el manejo, han intentado homogenizarse por medio de la capacitación de los profesionales de la salud que están en contacto con estos pacientes, con miras a evitar el subdiagnóstico de condiciones potencialmente fatales y el ingreso innecesario por causas que no requieren tratamiento urgente $\mathrm{e}^{22,23}$.

Así mismo, se han empleado pruebas de laboratorio como las transaminasas, posteriormente la creatin-kinasa y la fracción MB, hasta llegar a mediados del año 2000 al uso estandarizado de la troponina $T$ o I como estudio fundamental para el enfoque del síndrome coronario agudo sin elevación del segmento ST. Infortunadamente, la experiencia con los biomarcadores, sobretodo aquellos de alta sensibilidad, no es favorable. El enfoque actual en los servicios de urgencias se aleja de la historia clínica y el examen físico y se ha vuelto un biomarcador dependiente. A sabiendas de que el biomarcador ideal no existe, estas herramientas se deben usar de manera racional con el fin de tamizar, diagnosticar, estratificar el riesgo, definir el pronóstico e incluso determinar la duración y los efectos favorables o no de un tratamiento específico ${ }^{18,19}$.

El abordaje del dolor torácico en el servicio de urgencias no debe enforcarse sólo en el resultado de la troponina o ser "troponinocéntrico"'; de ahí que el personal encargado de la dirección del servicio de urgencias deba emprender e incentivar campañas que permitan orientar al clínico que es el primer respondedor ante los casos de dolor torácico para 
Tabla 3 Razón de prevalencia de los factores de riesgo para síndrome coronario agudo según los hallazgos angiográficos

\begin{tabular}{|c|c|c|c|c|c|c|}
\hline Factor de riesgo & Lesiones epicárdicas $(n=201)$ & Sin lesiones epicárdicas $(n=210)$ & RP & IC 95 & & Valor de $\mathrm{p}$ \\
\hline Hipertensión arterial, no. (\%) & $144(71,64)$ & $150(71,43)$ & 1,01 & 0,64 & 1,58 & 0,961 \\
\hline Diabetes mellitus, no. (\%) & $50(24,88)$ & $59(28,10)$ & 0,84 & 0,53 & 1,34 & 0,459 \\
\hline Dislipidemia, no. (\%) & $63(31,34)$ & $49(23,33)$ & 1,50 & 0,94 & 2,38 & 0,068 \\
\hline Tabaquismo, no. (\%) & $67(33,33)$ & $63(30,00)$ & 1,16 & 0,75 & 1,80 & 0,467 \\
\hline IAM previo, no. (\%) & $98(48,76)$ & $83(39,52)$ & 1,45 & 0,96 & 2,19 & 0,059 \\
\hline Angina, no. (\%) & $29(14,43)$ & $52(24,76)$ & 0,51 & 0,29 & 0,87 & 0,008 \\
\hline
\end{tabular}

que se efectúe un enfoque clínico adecuado y que, cuando se solicite un estudio diagnóstico se conozcan ampliamente su cinética y las implicaciones de su resultado con el fin de corroborar una sospecha clínica mas no generar una serie de diagnósticos diferenciales como su punto de partida.

Según la tercera definición de infarto agudo de miocardio, tanto de las guías europeas de cardiología como las norteamericanas, el diagnóstico de trabajo de síndrome coronario agudo depende de la clínica sugestiva de enfermedad coronaria, asociada a biomarcadores positivos con un incremento o descenso del $20 \%$, configurando el diagnóstico y por ende, haciendo imperativo el cateterismo cardiaco como método invasivo para estratificar la enfermedad y brindar tratamiento oportuno ${ }^{6-8,13}$.

Con base en los resultados de este estudio, corresponden a un grupo de pacientes que fueron sometidos a un estudio invasivo producto de una mala interpretación de un paraclínico, desconociendo las causas alternativas que podrían generar resultados falsos positivos. Al observar las posibles causas que ocasionan la elevación de la troponina de alta sensibilidad, la presencia de disfunción renal avanzada, crisis hipertensivas o incluso la existencia de enfermedad coronaria estable se infiere que su aumento, sin ser falsos positivos, no refleja enfermedad arterial oclusiva aguda. Para tratar de disminuir estas fallas en la interpretación, en las guías de práctica clínica europeas y colombianas se han trazado unos algoritmos que le permiten al clínico actuar desde el servicio de urgencias $^{3,15}$.

Durante el tiempo que se llevó a cabo este estudio se empleó la troponina de alta sensibilidad $\mathrm{T}$ de laboratorios Roche, cuyo punto de referencia para considerarse positivo es de $0,014 \mathrm{ng} / \mathrm{ml}$. Conscientes de esta información, el abordaje que se ha sugerido emplear requiere un análisis detallado con base en los siguientes puntos: paciente con factores de riesgo cardiovascular para enfermedad coronaria, características del dolor típicas, atípicas o no coronarias y existencia de alguna limitación previa por síntomas cardiovasculares durante la actividad física. En caso de corroborarse esta información y considerar un posible síndrome coronario agudo, el médico que aborda al paciente en el servicio de urgencias evaluará el electrocardiograma que, en caso de ser negativo no descartará la presencia de síndrome coronario agudo pero disminuirá la probabilidad pretest para el mismo ${ }^{3,10,15}$.

Posteriormente, si el paciente fue catalogado dentro de un síndrome coronario agudo, se solicitará el biomarcador consciente del tiempo de evolución, el tiempo de ingreso a la institución y la duración exacta del dolor para poder considerar que el estudio solicitado aporta valor predictivo, tanto negativo como positivo. Una vez se obtengan los resultados, estos se analizarán a la luz de las posibles causas que lo eleven que no sean de origen isquémico agudo (crisis hipertensiva, lesión renal, arritmia cardiaca) y se determinará si es positivo al límite superior de referencia o no. Si es negativo el resultado del biomarcador, se solicitará un control a las tres horas en el cual se observará un incremento del $50 \%$ para poder definir un síndrome coronario agudo. De ser positivo el valor inicial del biomarcador se pedirá un control y se observará un incremento del $20 \%$ para poder definir síndrome coronario agudo. Finalmente, cuando se haya analizado el caso a la luz de los datos previamente enunciados, se elegirá una estrategia de estratificación (emergencia, temprana o tardía) según las diferentes escalas de riesgo pronósticas $^{11,12,15}$.

De la muestra de pacientes estudiados, en quienes no se documentaron lesiones angiográficamente significativas en el cateterismo cardiaco, la troponina no cumplía los criterios de positividad mencionados previamente. Del grupo de pacientes con enfermedad coronaria angiográficamente significativa en cateterismo cardiaco, la combinación de tres o más factores de riesgo cardiovascular en presencia de biomarcador positivo se correlacionaba fuertemente con hallazgos en la arteriografía.

En los resultados del estudio realizado, es evidente el desconocimiento tanto para el uso como para su interpretación, dado que, de los resultados negativos un alto porcentaje no cumplía los criterios de positividad basados en un cambio porcentual mayor o igual al $20 \%$ independiente del resultado del primer biomarcador. Un subgrupo de pacientes fue llevado a estratificación invasiva a pesar de tener los biomarcadores negativos o incluso con posibles causas alternas que expliquen la elevación del biomarcador.

En lo concerniente a las limitaciones del estudio, no se cuenta con datos suficientes para determinar la correlación entre el cambio absoluto o porcentual de la troponina y la presencia de lesiones angiográficamente significativas. Tampoco se puede elaborar una directriz para orientar al clínico en el servicio de urgencias sobre cuándo, con qué factores de riesgo o con qué nivel de troponina se debe hacer el estudio para poder identificar lesiones durante el cateterismo cardiaco. Se requieren estudios adicionales con un tamaño de muestra mayor y sin tanta limitación en la disponiblidad de datos, además de implementar medidas para que desde el servicio de urgencias se optimice la atención y valoración de los pacientes con diagnóstico presuntivo de síndrome coronario agudo. 


\section{Conclusiones}

Durante el periodo de estudio se recolectó información de 411 pacientes con diagnóstico presuntivo de síndrome coronario agudo sin elevación del segmento ST que asistieron al servicio de urgencias, cuyas características son similares a las encontradas en publicaciones de registros internacionales.

Pese a que el dolor torácico fue típico en más de la mitad de los pacientes, el valor de troponina no cumplió los criterios de positividad en quienes no se documentaron lesiones angiográficamente significativas en el cateterismo cardiaco.

Se requieren registros y estudios descriptivos con un mayor número de pacientes para hacer una descripción más completa y determinar sí existen diferencias o no entre el cambio en el valor del biomarcador y la presencia de lesiones angiográficamente significativas.

\section{Conflicto de intereses}

Ninguno.

\section{Bibliografía}

1. Owens PL, Barrett ML, Gibson TB, Andrews RM, Weinick RM, Mutter RL. Emergency department care in the United States: a profile of national data sources. Ann Emerg Med. 2010;56:150-65.

2. Six AJ, Backus BE, Kelder JC. Chest pain in the emergency room: value of the HEART score. Neth Heart J Mon J Neth Soc Cardiol Neth Heart Found. 2008;16:191-6.

3. Beltran JR, Beltran R, Caicedo VM, Garcia M, Garcia E. Guías de síndrome coronario agudo sin elevación del segmento ST. Rev Colomb Cardiol. 2008;15:141-232.

4. Mendoza F. Dolor torácico en el servicio de urgencias: «un reto por enfrentar». Rev Colomb Cardiol. 2003;10:455-64.

5. Apple FS, Collinson PO. IFCC Task Force on Clinical Applications of Cardiac Biomarkers Analytical characteristics of highsensitivity cardiac troponin assays. Clin Chem. 2012;58:54-61.

6. Babuin L, Jaffe AS, Troponin:. the biomarker of choice for the detection of cardiac injury. CMAJ Can Med Assoc J J Assoc Medicale Can. 2005;173:1191-202.

7. Jaffe AS, Babuin L, Apple FS. Biomarkers in acute cardiac disease: the present and the future. J Am Coll Cardiol. 2006;48:1-11.

8. Thygesen K, Alpert JS, Jaffe AS, Simoons ML, Chaitman BR, White HD, et al. Third universal definition of myocardial infarction. Circulation. 2012;126:2020-35.

9. French JK, White HD. Clinical implications of the new definition of myocardial infarction. Heart Br Card Soc. 2004;90:99-106.

10. Bakshi TK, Choo MKF, Edwards CC, Scott AG, Hart HH, Armstrong GP. Causes of elevated troponin I with a normal coronary angiogram. Intern Med J. 2002;32:520-5.
11. le EHY, Klootwijk PJ, Weimar W, Zietse R. Significance of acute versus chronic troponin T elevation in dialysis patients. Nephron Clin Pract. 2004;98:c87-92.

12. Reichlin T, Irfan A, Twerenbold R, Reiter M, Hochholzer W, Burkhalter $\mathrm{H}$, et al. Utility of absolute and relative changes in cardiac troponin concentrations in the early diagnosis of acute myocardial infarction. Circulation. 2011;124:136-45.

13. Thygesen K, Mair J, Katus H, Plebani M, Venge P, Collinson $\mathrm{P}$, et al. Recommendations for the use of cardiac troponin measurement in acute cardiac care. Eur Heart J. 2010;31: 2197-204.

14. Wallace TW, Abdullah SM, Drazner MH, Das SR, Khera A, McGuire DK, et al. Prevalence and determinants of troponin $T$ elevation in the general population. Circulation. 2006;113: 1958-65.

15. Roffi M, Patrono C, Collet J-P, Mueller C, Valgimigli M, Andreotti F, et al. 2015 ESC Guidelines for the management of acute coronary syndromes in patients presenting without persistent ST-segment elevation: Task Force for the Management of Acute Coronary Syndromes in Patients Presenting without Persistent ST-Segment Elevation of the European Society of Cardiology (ESC). Eur Heart J. 2016;37:267-315.

16. Wu AHB, Jaffe AS. The clinical need for high-sensitivity cardiac troponin assays for acute coronary syndromes and the role for serial testing. Am Heart. 2008;155:208-14.

17. Zhelev Z, Hyde C, Youngman E, Rogers M, Fleming S, Slade T, et al. Diagnostic accuracy of single baseline measurement of Elecsys Troponin T high-sensitive assay for diagnosis of acute myocardial infarction in emergency department: systematic review and meta-analysis. BMJ. 2015;350:h15.

18. Ahmed W, Schlett CL, Uthamalingam S, Truong QA, Koenig W, Rogers IS, et al. Single resting hsTnT level predicts abnormal myocardial stress test in acute chest pain patients with normal initial standard troponin. JACC Cardiovasc Imaging. 2013;6:72-82.

19. de Lemos JA, Drazner MH, Omland T, Ayers CR, Khera A, Rohatgi $A$, et al. Association of troponin $T$ detected with a highly sensitive assay and cardiac structure and mortality risk in the general population. JAMA. 2010;304:2503-12.

20. Reichlin T, Hochholzer W, Bassetti S, Steuer S, Stelzig C, Hartwiger S, et al. Early diagnosis of myocardial infarction with sensitive cardiac troponin assays. N Engl J Med. 2009;361:858-67.

21. Reichlin T, Schindler C, Drexler B, Twerenbold R, Reiter M, Zellweger $\mathrm{C}$, et al. One-hour rule-out and rule-in of acute myocardial infarction using high-sensitivity cardiac troponin T. Arch Intern Med. 2012;172:1211-8.

22. Hoyo FD, del, Fernández MJ, Casasnovas IS. Dolor torácico en Urgencias. Med - Programa Form Médica Contin Acreditado. 2015;11:5167-74.

23. Pickering JW, Than MP, Cullen L, Aldous S, Ter Avest E, Body R, et al. Rapid Rule-out of Acute Myocardial Infarction With a Single High-Sensitivity Cardiac Troponin T Measurement Below the Limit of Detection: A Collaborative Meta-analysis. Ann Intern Med. 2017. 